\title{
Persisting Meissner state and incommensurate phases of hard-core boson ladders in a flux
}

\author{
M. Di Dio, ${ }^{1}$ S. De Palo,,${ }^{1,2}$ E. Orignac, ${ }^{3}$ R. Citro, ${ }^{4}$ and M.-L. Chiofalo ${ }^{5}$ \\ ${ }^{1}$ CNR-IOM-Democritos National Simulation Centre, UDS Via Bonomea 265, I-34136 Trieste, Italy \\ ${ }^{2}$ Dipartimento di Fisica Teorica, Università di Trieste, Trieste, Italy \\ ${ }^{3}$ Laboratoire de Physique de l'École Normale Supérieure de Lyon, CNRS UMR5672, 46 Allée d'Italie, F-69364 Lyon Cedex 7, France \\ ${ }^{4}$ Dipartimento di Fisica “E.R. Caianiello," Università degli Studi di Salerno and Unità Spin-CNR, \\ Via Giovanni Paolo II, 132, I-84084 Fisciano (Sa), Italy \\ ${ }^{5}$ Dipartamento di Fisica “Enrico Fermi” "and INFN, Università di Pisa, Largo Bruno Pontecorvo 3, I-56127 Pisa, Italy
}

(Received 11 June 2015; revised manuscript received 13 July 2015; published 26 August 2015)

\begin{abstract}
The phase diagram of a half-filled hard-core boson two-leg ladder in a flux is investigated by means of numerical simulations based on the density matrix renormalization group (DMRG) algorithm and bosonization. We calculate experimentally accessible observables such as the momentum distribution, as well as rung current, density wave, and bond-order wave correlation functions, allowing us to identify the Mott Meissner and Mott vortex states. We follow the transition from commensurate Meissner to incommensurate vortex state at increasing interchain hopping until the critical value [Piraud et al. Phys. Rev. B 91, 140406 (2015)] above which the Meissner state is stable at any flux. For flux close to $\pi$, and below the critical hopping, we observe the formation of a second incommensuration in the Mott vortex state that could be detectable in current experiments.
\end{abstract}

DOI: 10.1103/PhysRevB.92.060506

PACS number(s): 67.85.-d, 03.75.Lm, 05.30.Rt, 64.70.Rh

Superconductors in external magnetic field $H<H_{c 1}$ exhibit the Meissner-Ochsenfeld effect where surface currents screen completely the magnetic field in the bulk, resulting in perfect diamagnetism [1]. Type-I superconductors return to the normal state for $H>H_{c 1}$, while in type-II superconductors, for $H_{c 1}<H<H_{c 2}$ a vortex phase is formed, in which the magnetic field partially penetrates the system along flux lines surrounded by screening currents. This behavior can be understood in the framework of spontaneous breaking of a global U(1) symmetry via the Landau-Ginzburg equation [1]. In a quasi-one-dimensional system, such symmetry breaking is precluded by the Mermin-Wagner-Hohenberg theorem [2,3]. However, in the case of a bosonic two-leg ladder [4-6], an ana$\log$ of the Meissner phase was predicted to exist in the ground state for low flux, while for higher flux a Tomonaga-Luttinger liquid (TLL) of vortices was expected. The quantum phase transition between these two states is in the commensurateincommensurate (C-IC) universality class [7,8]. Other orderings have been predicted, such as chiral superfluid order at half a flux quantum per plaquette $[4,9,10]$ and a chiral Mott insulating phase [11-15], which is a Mott regime [16] possessing chiral currents as well as a spin-density-wave phase. DMRG studies of ladders with diagonal interchain hopping are also available [17-22]. While the original proposal was made in the context of Josephson junction ladders, where the quantum effects are spoiled by dissipation [23], the advent of ultracold atomic gases offers another realization of strongly interacting one-dimensional boson systems [24,25]. Moreover, it has been shown theoretically [26,27] and experimentally [28] how artificial gauge field could be created in these systems. Recently, the transition from Meissner to vortex phases in noninteracting bosonic ladders of ultracold atoms has been studied experimentally at fixed flux $\pi / 2$ per plaquette and variable interleg hopping [29].

In this Rapid Communication we explore the phase diagram of hard-core spinless bosons on a two-leg ladder at half-filling as a function of flux and interchain hopping by means of numerical simulations using the DMRG algorithm and bosonization. We find, in agreement with Ref. [30], that hard-core constraints cause a significant enlargement of the Meissner phase over the vortex one with respect to the noninteracting case: above a critical value of the interchain hopping [30] the system remains in the Mott-Meissner (MM) state for any flux (see Fig. 1). Below the critical interchain hopping, both the behavior of the momentum distribution and of the rung current, show that the transition from a MM to a Mott-vortex (MV) state falls in the universality class of the C-IC transition [5]. For fluxes close to $\pi$, we observe another incommensuration, whose origin is discussed within bosonization.

We consider [5] hard-core spinless bosons on a two-leg ladder, with a flux per plaquette $\lambda$ and interchain hopping $\Omega$ :

$$
\begin{aligned}
H_{\lambda}= & -t \sum_{j, \sigma}\left(b_{j, \sigma}^{\dagger} e^{i \lambda \sigma} b_{j+1, \sigma}+\text { H.c. }\right) \\
& +\Omega \sum_{j}\left(b_{j, \uparrow}^{\dagger} b_{j, \downarrow}+\text { H.c. }\right),
\end{aligned}
$$

with $b_{j, \sigma}^{\dagger}\left(b_{j, \sigma}\right)$ bosonic creation (annihilation) operator at site $j, \sigma= \pm 1 / 2$ the chain index, and $t e^{i \lambda \sigma}$ the hopping amplitude along the chain $\sigma$. This Hamiltonian can be mapped onto a system of spin-1/2 bosons [31] with spin-orbit coupling in a transverse magnetic field [32] with each spinor state corresponding to one leg of the ladder. For half-filling, i.e., for one boson per rung, at $\lambda=0$ and $\Omega \neq 0$ the ground state of (1) is a rung-Mott insulator [33]. For $\lambda>0$, according to the bosonization treatment [34], two phases with a total density gap are expected [5,13-15], the MM and the MV state. In the MM state, for $0<\lambda<\lambda_{c}$, two currents of opposite sign flow along the legs [30]; the interchain current

$$
J_{r}(l)=i \Omega\left(b_{l, \uparrow}^{\dagger} b_{l, \downarrow}-b_{l, \downarrow}^{\dagger} b_{l, \uparrow}\right)
$$

has zero expectation value and exponentially decaying correlations, and the screening current, i.e., the difference between 

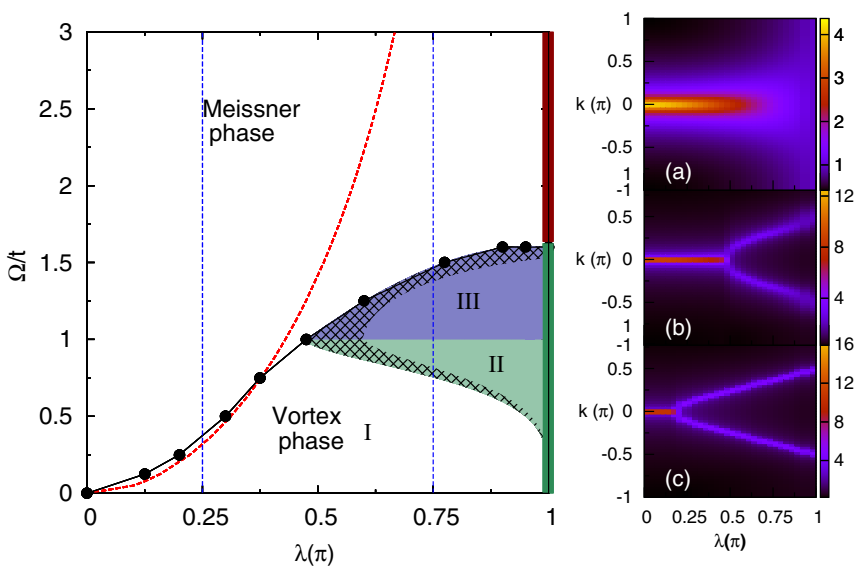

FIG. 1. (Color online) Left panel: Phase diagram of (1) as a function of flux per plaquette $\lambda$ and $\Omega / t$. The phase boundary between the Meissner and the vortex phase is shown by the black solid line, displaying the persistence of the Meissner phase [30] above the threshold $\Omega>\Omega_{c}$ for all fluxes $\lambda$, except $\lambda=\pi$. For comparison the red-dashed line shows the boundary $\lambda_{c}^{(0)}(\Omega)$ for the noninteracting system. In the shaded area a second incommensuration appears. In the green area (region II) extra peaks at $k=\pi$ develop in the FT of the rung current correlations and they become the dominant correlations in the blue region (III). The double line (green vs dark red) at $\lambda=\pi$ represents the transition to a localized phase. In the right panel we show intensity plots of $n(k)$ versus $\lambda$ and $k$. In panel (a), at $\Omega / t=1.75$ the system is always in the MM phase; indeed, only a single maximum at $k=0$ is visible for all $\lambda$. At $\lambda=\pi, n(k)=1$ indicating the formation of a fully localized state (dark red solid line). In panel (b) and (c), for $\Omega / t=1$ and 0.25 , respectively, the transition from MM to the MV with two maxima symmetric around $k=0$ is shown.

the currents of the two legs

$$
J_{s}=-i t \sum_{j, \sigma}\left(\sigma e^{i \lambda \sigma} b_{j, \sigma}^{\dagger} b_{j+1, \sigma}-\sigma e^{-i \lambda \sigma} b_{j+1, \sigma}^{\dagger} b_{j, \sigma}\right),
$$

is a smooth function of the applied flux (increasing linearly at small flux). On increasing the flux $\lambda>\lambda_{c}(\Omega)$, the system enters the MV state; there is a sudden drop [30] of the screening current $J_{s}$ and simultaneously the rung current correlations decay becomes algebraic [30] with an incommensurate modulation of wave vector $q(\lambda)$. Close to the transition point $\lambda_{c}(\Omega)$, the wave vector $q(\lambda) \sim \sqrt{\lambda^{2}-\lambda_{c}^{2}}$. In the noninteracting case, the Hamiltonian equation (1) can be readily diagonalized $[14,16]$ and $\lambda_{c}^{(0)}(\Omega)=2 \arctan [\Omega /(4 t)]$. The occurrence of the MV phase can be seen out also in the total, as well as in the spin resolved momentum distribution [6] of the system:

$$
n(k)=\sum_{\sigma} n_{\sigma}(k)=\frac{1}{L} \sum_{\sigma} \sum_{i, j}^{L-1} e^{i k\left(r_{i}-r_{j}\right)}\left\langle b_{i, \sigma}^{\dagger} b_{j, \sigma}\right\rangle .
$$

In the MM phase $n(k)$ has a single maximum at $k=0$, whereas in the MV phase it exhibits a pair of maxima $k= \pm q(\lambda) / 2$ [35]. We have obtained the ground-state phase diagram of (1) by computing various observables such as the momentum distribution and the screening current $J_{s}$ together with the
Fourier Transform (FT) $C(k)=\sum_{l} e^{-i k l}\left\langle J_{r}(l) J_{r}(0)\right\rangle$ of the rung current correlation function.

While performing simulations with both periodic (PBC) and open boundary conditions, we found the former to be more suitable for our system, despite the well-known computationally more demanding convergence properties typical of PBC [36-38]. As such we run simulations employing PBC for system sizes ranging from $L=16$ to $L=64$, keeping up to $m=1256$ states during the renormalization procedure. In this way the truncation error, i.e., the weight of the discarded states, is at most of order $10^{-6}$, while the maximum error on the ground-state energy is of order $5 \times 10^{-5}$ at its most. We further extrapolate in the limit $m \rightarrow \infty$ all the quantities calculated to characterize the phase diagram.

In Fig. 1, we summarize our findings for the phase diagram at half-filling. At variance with the noninteracting case where there is a critical $\lambda_{c}^{(0)}(\Omega)$ for all $\Omega$, in the presence of the hard-core interaction, for interchain hoppings $\Omega>\Omega_{c}$, the commensurate-incommensurate transition disappears [30] and the MM phase is stable for all fluxes. Another effect of the hard-core interaction, as we will discuss below, is that in the vortex phase, at $\lambda=\pi$ and $\lambda$ close to $\pi$, a commensurate peak appears in $C(k \simeq \pi)$, along with an incommensuration in the density correlations. At $\lambda=\pi$, and for $\Omega>\Omega_{c}$ a fully rung localized phase is obtained. Such rung localized ground state was discussed in the limit $\Omega \gg t$ in Ref. [30].

We have characterized the nature of the MM and MV phases by examining $C(k)$, the staggered boson density wave $S(k)$ and the symmetric bond-order wave $S_{\text {BOw }}^{c}$ static structure factors which bring information on the spin-density and bond-order waves, respectively:

$$
\begin{gathered}
S(k)=\frac{1}{L} \sum_{\substack{j, l=0 \\
\sigma \sigma^{\prime}}}^{L-1} e^{i k(j-l)} \operatorname{sgn}\left(\sigma \sigma^{\prime}\right)\left\langle n_{j, \sigma} n_{l \sigma^{\prime}}\right\rangle, \\
S_{\mathrm{BOW}}^{c}(k)=\frac{1}{L} \sum_{j, l=0}^{L-1} e^{i k(j-l)}\left\langle\delta B_{j} \delta B_{l}\right\rangle,
\end{gathered}
$$

where $B_{j}=\sum_{\sigma} b_{j+1, \sigma}^{\dagger} b_{j, \sigma}+$ H.c. and $\delta B_{j}=B_{j}-\left\langle B_{j}\right\rangle$.

In Fig. 2 we follow the the MM-MV phase transition at small $\lambda$ and $\Omega$ (see cut one in Fig. 1). As predicted from bosonization [32] the vortex phase is signaled by the appearance in $C(k)$ of two cusplike peaks respectively at $k=q(\lambda)$ and $k=2 \pi-q(\lambda)$ [see panel (a) of Fig. 2] whose heights do not scale with the size of the system [see Fig. 1 of Ref. [32]]. In MV phase, the spin resolved momentum distribution $n_{\sigma}(k)$ shows a symmetric peak centered at $k=\sigma q(\lambda)$, as predicted by bosonization. In this region of parameter space the correlation length associated with the Mott gap [33] is comparable with the system size, and the peak takes a cusplike shape as in a Tomonaga-Luttinger liquid [31], instead of the typical Lorentzian shape expected for a Mott insulator. Also $S(k)$ shows the expected low momentum behavior according to bosonization approach: in the MM phase $S(k)=S(0)+a k^{2}+o\left(k^{2}\right)$, with $S(0)>0$, while in the MV phase $S(k)=\frac{K_{s}^{*}|k|}{\pi}+o(k)$, with $K_{s}^{*}=1$ (as expected for a hard-core boson system) a signature of a TLL of vortices. The transition is also seen in $S(k \simeq \pi)$. In the MM phase, $S(k \simeq \pi)$ shows a Lorentzian-shaped peak, while in the MV 

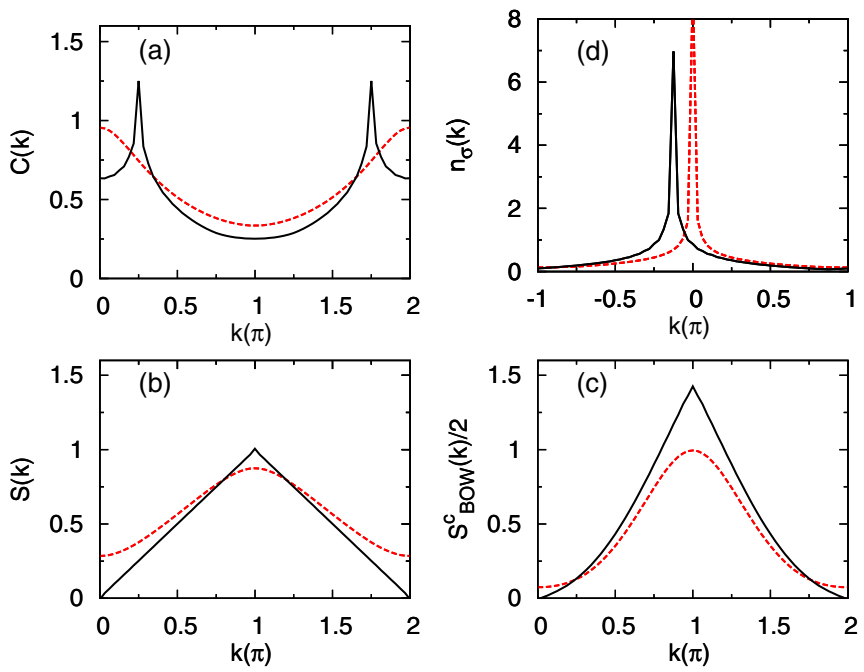

FIG. 2. (Color online) We show FT of correlation functions as from DMRG simulation for $L=64$ at $\lambda=\pi / 4$ for two different values of the $\Omega / t=0.0625$ and 1 , respectively, in the vortex (black solid line) and Meissner phases [dashed (red) line]. Panel (a) shows the FT of the rung-current correlation function $C(k)$, panel (b) the spin correlation functions $S(k)$, and panel (c) the charge bond-order correlation function $S_{\mathrm{BOW}}^{c}$. In panel (d) the spin resolved momentum distribution is shown, with $n_{-\sigma}(k)=n_{\sigma}(-k)$.

phase this peak takes a cusplike shape. Density correlation functions such as $S(k)$ are experimentally accessible using light scattering techniques [39]. A similar change across the MM-MV transition is also seen in the correlation function $S_{\mathrm{BOW}}^{c}(k \simeq \pi)$ (see Supplemental Material [32]). This description breaks down when $\lambda$ is no longer a small quantity as $q(\lambda)$ would be comparable to the momentum cutoff.

At $\lambda=\pi$ the major changes from the conventional C-IC transition at small flux are observed. To derive the low energy Hamiltonian it becomes necessary to choose the gauge with the vector potential along the rungs of the ladder, so that the interchain hopping reads

$$
H_{\text {hop. }}=\Omega \sum_{j, \sigma}(-)^{j} b_{j, \sigma}^{\dagger} b_{j,-\sigma} .
$$

After applying bosonization, the hopping Hamiltonian can be rewritten in terms of a free boson $\phi_{c}$ describing the total density fluctuations coupled to SU(2), Wess-Zumino-Novikov-Witten currents $\mathbf{J}_{R, L}$ describing the chain antisymmetric density fluctuations by a term $\propto \Omega \cos \sqrt{2} \phi_{c}\left(J_{R}^{y}+J_{L}^{y}\right)$ (see Ref. [32] for details). Such a term can be treated in mean-field theory [40-43]. This procedure leads to an effective Hamiltonian with a gap $\Delta_{c} \sim \Omega^{2}$ for the total density excitations, while the antisymmetric density modes remain gapless and develop an incommensuration of wave vector $p(\Omega) \propto \Omega^{2}$ (see Fig. 2 in Ref. [32]). The presence of this predicted incommensuration is visible in the low momentum behavior of $C(k)$ and $S(k)$ [panels (a) and (b), respectively, in Fig. 3] that become $\propto \frac{K_{s}^{*}}{2 \pi}(|k-p(\Omega)|+|k+p(\Omega)|)$, i.e., constant for $|k|<p(\Omega)$ and linear in $k$ for $|k|>p(\Omega)$. In the $S_{\mathrm{BOW}}^{c}(k)$ we observe a cusp at the same vectors $p(\Omega)$ [panel (c) of Fig. 3]. As expected, all these correlation functions also develop a peak
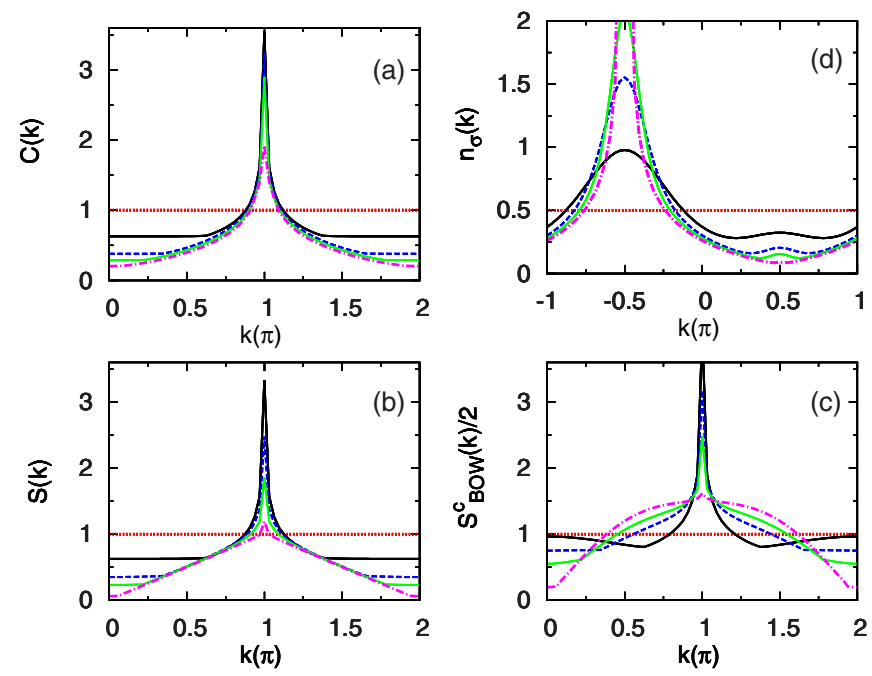

FIG. 3. (Color online) We show FT of correlation functions as from DMRG simulations for $L=64$ at $\lambda=\pi$ at various $\Omega / t$. Panel (a) shows the FT of the rung-current correlation function $C(k)$, panel (b) the spin correlation function $S(k)$, and panel (c) the charge bond-order correlation function $S_{\text {BOw }}^{c}$. In panel (d) the spin resolved momentum distribution is shown. Dotted (red) curves are for $\Omega / t=1.75$ in the fully localized state, while black solid, dashed (blue), gray (green) solid, and dot-dashed (magenta) lines are, respectively, for $\Omega / t=1.5,1.25,1$, and 0.5 .

at $k=\pi / a$, where $a$ is the lattice spacing. A sign of the incommensurability at $\lambda=\pi$ should be visible also in the momentum distribution $n_{\sigma}(k)$ [see Fig. 3, panel (d)]. In this case, a calculation based on non-Abelian bosonization and operator product expansion, would lead to three Lorentzianlike peaks centered in $\pi /(2 a)$ and $\pi /(2 a) \pm p(\Omega) / 2$. However, these peaks cannot be separated if the correlation length in real space $u_{c} / \Delta_{c} \sim \Omega^{-2}$ is shorter than the wavelength $2 \pi / p(\Omega) \sim \Omega^{-2}$. In the numerical simulations, at $L=64$ in PBC (see Fig. 3) a broad peak is observed for $k=\frac{\pi}{2 a}$.

When $\lambda \lesssim \pi$ (second cut in Fig. 1) we can proceed analogously to the previous case and choose a gauge such that

$$
\begin{aligned}
H= & -t \sum_{j, \sigma}\left(b_{j, \sigma}^{\dagger} e^{i(\lambda-\pi) \sigma} b_{j+1, \sigma}+\text { H.c. }\right) \\
& +\Omega \sum_{j, \sigma}(-1)^{j} b_{j, \sigma}^{\dagger} b_{j,-\sigma},
\end{aligned}
$$

and define $\delta \lambda=(\lambda-\pi)$, so that the bosonized Hamiltonian contains the extra term $\delta \lambda\left(J_{R}^{z}-J_{L}^{z}\right)$. For this case, the Fourier transform of the rung current correlation will present peaks at $k=\frac{\pi}{a}$ and $k=\frac{\pi}{a} \pm \sqrt{p(\Omega)^{2}+(\delta \lambda / a)^{2}}$. When $\delta \lambda$ is increased, these last two peaks become dominant, and we cross over to the behavior already discussed for weak $\lambda$. At $\lambda<\pi$ the $C(k)$ (see Fig. 4) shows, beside the peak at $k=\frac{\pi}{a}$, two peaks symmetric around $k=\frac{\pi}{a}$; in real space these last two oscillations exhibit an exponential decay for $\Omega / t>1$ and a power law for $\Omega / t \leqslant 1$ (regions III and II in Fig. 1). The situation is reversed for the oscillation at $k=\frac{\pi}{a}$. At $\Omega / t=1$ all oscillations, for systems with $L=64$ in $\mathrm{PBC}$, exhibit power law decay. The effect of this incommensuration can also be 

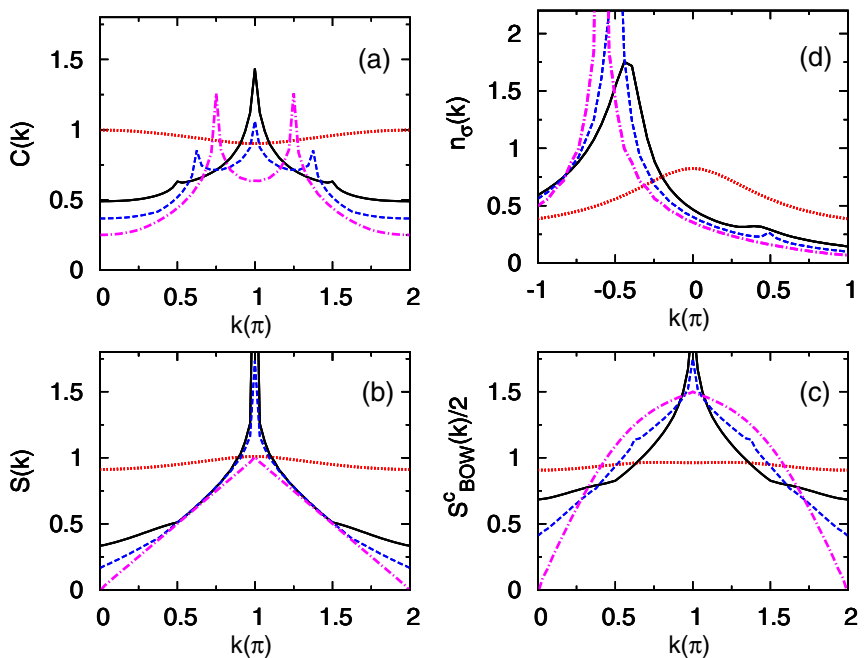

FIG. 4. (Color online) We show FT of correlation functions as from DMRG simulations for $L=64$ at $\lambda=3 \pi / 4$ at various $\Omega / t$. Panel (a) shows the FT of the rung-current correlation function $C(k)$, panel (b) the spin correlation function $S(k)$, and panel (c) the symmetric bond-order correlation function $S_{\mathrm{BOw}}^{c}$. In panel (d) the chain resolved momentum distribution is shown, with $n_{-\sigma}(k)=$ $n_{\sigma}(-k)$. Dotted (red) curves are for $\Omega / t=1.5$ in the Meissner phase, while black solid, dashed (blue), and dot-dashed (magenta) lines are, respectively, for $\Omega / t=1.25,1$, and 0.625 .

followed in the behavior at small $k$ of $S(k)$ that instead of being a constant value for $k<\sqrt{p(\Omega)^{2}+(\delta \lambda / a)^{2}}$ shows a linear behavior. In $S_{\mathrm{BOW}}^{c}(k)$, for $\Omega / t \leqslant 1$ two symmetric peaks are present at $k= \pm q(\lambda)$. We checked that the phase is a single component Tomonaga-Luttinger liquid by computing the von Neumann entropy at $\Omega / t=1.5$ for $\lambda=0.75 \pi$ and $0.8125 \pi$ and obtaining the expected logarithmic dependence with system size [30], ruling out a chiral Mott insulator [13,15] for $\lambda \lesssim \pi$. At general commensurate filling $n$ and flux $\lambda=2 \pi n$, the incommensuration generating term becomes $-i \Omega e^{i \sqrt{2} \phi_{c}}\left(J_{R}^{+}+J_{L}^{-}\right)+$H.c., leading to density wave phases with incommensuration. Let us finish by noting that such incommensuration is specific of hard-core boson systems. With less repulsive interactions, the term that gives rise to the vortex lattice state would be relevant [13,15,44], while stronger repulsion would make the term stabilizing the checkerboard density wave relevant. Adding a nearest neighbor intrachain interaction $V$ to the hard-core repulsion, a first phase transition at $V<0$ will separate the vortex lattice from the incommensurate state, and a second transition at $V>0$ will separate the incommensurate state from the density wave state.

In conclusion, we have studied a two-leg hard-core boson ladder in an artificial gauge field. In contrast to the noninteracting case, the vortex phase is suppressed when the interchain hopping exceeds a threshold value, as found in Ref. [30]. At flux $\pi$ per plaquette and $\Omega / t>1.6$ the ground state becomes a tensor product of singly occupied rungs, as was expected [30] in the $\Omega / t \rightarrow \infty$ limit. For $\Omega / t<1.6$, we have obtained an incommensurate insulating state similar to the spin-nematic state of frustrated $X X Z$ spin chains [40-43]. In the case of a system of weakly coupled ladders, a long range ordered phase could form in which density wave or rung current would possess a long range commensurate order, but exponentially damped incommensurate correlations would still be present. The presented results could be detectable in current experiments with cold atoms [29] and the evidence of a persisting Meissner state could be relevant for quantum computing purposes in defining a stable flux qubit $[45,46]$.

We thank F. Ortolani for the DMRG code. Simulations were run at Università di Salerno and Università di Pisa local computing facilities. The authors acknowledge partial support from PRIN-2011 "Collective Quantum phenomena: From strongly correlated system to quantum simulators."
[1] M. Tinkham, Introduction to Superconductivity (McGraw-Hill, New York, 1975).

[2] N. D. Mermin and H. Wagner, Phys. Rev. Lett. 17, 1133 (1967).

[3] P. C. Hohenberg, Phys. Rev. 158, 383 (1967).

[4] M. Kardar, Phys. Rev. B 33, 3125 (1986).

[5] E. Orignac and T. Giamarchi, Phys. Rev. B 64, 144515 (2001).

[6] M.-C. Cha and J.-G. Shin, Phys. Rev. A 83, 055602 (2011).

[7] G. I. Dzhaparidze and A. A. Nersesyan, Pis'ma Zh. Eksp. Teor. Phys. 27, 356 (1978) [Sov. Phys. JETP Lett. 27, 334 (1978)].

[8] V. L. Pokrovsky and A. L. Talapov, Phys. Rev. Lett. 42, 65 (1979).

[9] E. Granato, Phys. Rev. B 42, 4797 (1990).

[10] Y. Nishiyama, Eur. Phys. J. B 17, 295 (2000).

[11] A. Dhar, M. Maji, T. Mishra, R. V. Pai, S. Mukerjee, and A. Paramekanti, Phys. Rev. A 85, 041602 (2012).

[12] A. Dhar, T. Mishra, M. Maji, R. V. Pai, S. Mukerjee, and A. Paramekanti, Phys. Rev. B 87, 174501 (2013).

[13] A. Petrescu and K. Le Hur, Phys. Rev. Lett. 111, 150601 (2013).
[14] A. Tokuno and A. Georges, New J. Phys. 16, 073005 (2014).

[15] A. Petrescu and K. Le Hur, Phys. Rev. B 91, 054520 (2015).

[16] A. Keleş and M. O. Oktel, Phys. Rev. A 91, 013629 (2015).

[17] J. Zhao, S. Hu, J. Chang, P. Zhang, and X. Wang, Phys. Rev. A 89, 043611 (2014).

[18] J. Zhao, S. Hu, J. Chang, F. Zheng, P. Zhang, and X. Wang, Phys. Rev. B 90, 085117 (2014).

[19] Z. Xu, W. S. Cole, and S. Zhang, Phys. Rev. A 89, 051604(R) (2014).

[20] S. Peotta, L. Mazza, E. Vicari, M. Polini, R. Fazio, and D. Rossini, J. Stat. Mech.: Theor. Exp. (2014) P09005.

[21] M. Piraud, Z. Cai, I. P. McCulloch, and U. Schollwöck, Phys. Rev. A 89, 063618 (2014).

[22] L. Barbiero, M. Abad, and A. Recati, arXiv:1403.4185.

[23] R. Fazio and H. van der Zant, Phys. Rep. 355, 235 (2001).

[24] B. Paredes, A. Widera, V. Murg, O. Mandel, S. Folling, I. Cirac, G. Shlyapnikov, T. Hansch, and I. Bloch, Nature (London) 429, 277 (2004). 
[25] T. Kinoshita, T. Wenger, and D. Weiss, Science 305, 5687 (2004).

[26] K. Osterloh, M. Baig, L. Santos, P. Zoller, and M. Lewenstein, Phys. Rev. Lett. 95, 010403 (2005).

[27] J. Ruseckas, G. Juzeliūnas, P. Öhberg, and M. Fleischhauer, Phys. Rev. Lett. 95, 010404 (2005).

[28] Y. Lin, K. Jimenez-Garcia, and I. B. Spielman, Nature (London) 471, 83 (2011).

[29] M. Atala, M. Aidelsburger, M. Lohse, J. Barreiro, B. Paredes, and I. Bloch, Nat. Phys. 10, 588 (2014).

[30] M. Piraud, F. Heidrich-Meisner, I. P. McCulloch, S. Greschner, T. Vekua, and U. Schollwöck, Phys. Rev. B 91, 140406 (2015).

[31] M. A. Cazalilla, R. Citro, T. Giamarchi, E. Orignac, and M. Rigol, Rev. Mod. Phys. 83, 1405 (2011).

[32] See Supplemental Material at http://link.aps.org/supplemental/ 10.1103/PhysRevB.92.060506 for a detailed bosonization calculation.

[33] F. Crépin, N. Laflorencie, G. Roux, and P. Simon, Phys. Rev. B 84, 054517 (2011).

[34] F. D. M. Haldane, Phys. Rev. Lett. 47, 1840 (1981).
[35] Note that the momentum distribution is a gauge dependent quantity, and that our gauge choice differs from that of Ref. [6].

[36] S. R. White, Phys. Rev. Lett. 69, 2863 (1992).

[37] S. R. White, Phys. Rev. B 48, 10345 (1993).

[38] U. Schollwöck, Rev. Mod. Phys. 77, 259 (2005).

[39] D. Clément, N. Fabbri, L. Fallani, C. Fort, and M. Inguscio, Phys. Rev. Lett. 102, 155301 (2009).

[40] A. A. Nersesyan, A. O. Gogolin, and F. H. L. Essler, Phys. Rev. Lett. 81, 910 (1998).

[41] P. Lecheminant, T. Jolicoeur, and P. Azaria, Phys. Rev. B 63, 174426 (2001).

[42] T. Jolicoeur and P. Lecheminant, Prog. Theor. Phys. Suppl. 145, 23 (2002).

[43] M. Zarea, M. Fabrizio, and A. Nersesyan, Eur. Phys. J. B 39, 155 (2004).

[44] S. Greschner, M. Piraud, F. Heidrich-Meisner, I. P. McCulloch, U. Schollwöck, and T. Vekua, arXiv:1504.06564.

[45] M. H. Devoret, A. Wallraff, and J. M. Martinis, arXiv:condmat/0411174.

[46] J. Q. You and F. Nori, Phys. Today 58(11), 42 (2005). 